\title{
Function and mode of action of cytohesins in the epidermal growth factor pathway in colorectal cancer cells
}

\author{
TAO PAN* , JUNFENG SUN*, JUN ZHOU, ZHIXUAN FU, YIWANG HU, SHU ZHENG and SUZHAN ZHANG \\ Cancer Institute, The Second Affiliated Hospital, Zhejiang University School of Medicine, \\ Hangzhou, Zhejiang 310009, P.R. China
}

Received August 15, 2012; Accepted November 15, 2012

DOI: $10.3892 / \mathrm{ol} .2012 .1064$

\begin{abstract}
Cytohesins have been identified as cytoplasmic ErbB receptor activators in certain cancers, exhibiting an important role in ErbB signaling. However, whether cytohesins are essential in colorectal cancer is unknown. The aim of the present study was to investigate whether cytohesins contribute to the epidermal growth factor (EGF) pathway in colorectal cancer cells. RT-PCR and immunofluorescence experiments were employed to detect the expression of cytohesins in colorectal cancer cell lines. The EGF pathway activation conditions were investigated by examining the phosphorylation of the epidermal growth factor receptor (EGFR) and intracellular signal-related kinases, with or without chemical inhibition (SecinH3) and knockdown of cytohesins. An MTT assay was conducted to examine the inhibitory effect of SecinH3 and cytohesin-specific siRNA in HT-29 cells. Results demonstrated that the four homologous members of the cytohesin family were expressed in the four colorectal cancer cell lines. Notably, a significantly higher expression level of cytohesin-2 (ARNO) compared with the other three homologous family members was observed. Stimulation with EGF and SecinH3, as well as knockdown of ARNO, are capable of reducing EGF pathway activation and proliferation of HT-29 cells. In conclusion, cytohesins play an essential role in the activation of the EGF pathway and may be a potential target in colorectal cancer therapy.
\end{abstract}

Correspondence to: Professor Suzhan Zhang, Cancer Institute (Key Laboratory of Cancer Prevention and Intervention, China National Ministry of Education, Key Laboratory of Molecular Biology in Medical Sciences), The Second Affiliated Hospital, Zhejiang University School of Medicine, 88 Jiefang Street, Hangzhou, Zhejiang 310009, P.R. China

E-mail: zhangscy1@126.com

*Contributed equally

Key words: epidermal growth factor receptor, cytohesin, colorectal cancer, pathway, ARNO

\section{Introduction}

Colorectal cancer is one of the most common clinical gastrointestinal cancers that poses a serious threat to human health. The GLOBOCAN 2008 estimates stated that colorectal cancer is the third most commonly diagnosed cancer in males and the second in females, with over 1.2 million new cancer cases and 608,700 fatalities occurring every year (1). Effective treatments for colorectal cancer include surgery, chemotherapy and targeted therapy. Chemotherapy and targeted therapy are the final strategies implemented to extend patient survival, particularly with advanced metastatic colorectal cancer $(2,3)$. However, present evidence-based medicine indicates that colorectal cancer patients hardly benefit from chemotherapy due to chemical toxicity and self-resistance. Therefore, targeted therapy has a greater potential and is currently being investigated further, with a greater research emphasis on cancer therapeutics. Throughout the past decade, targeted treatment of cancer has mainly focused on the epidermal growth factor (EGF) pathway $(4,5)$.

The epidermal growth factor receptor (EGFR) is a key member of the ErbB family, which consists of four members: EGFR (ErbB1), Her2 (ErbB2), Her3 (ErbB3) and ErbB4. In cancer cells, the extracellular domain of the EGFR binds to the EGF and the EGF pathway is activated; signaling is initiated to regulate the differentiation, survival, proliferation and migration of cancer cells (6). However, activation of the EGFR is required for signaling initiation; the ligand-induced conformational change in the receptor ectodomains results in the association of the cytoplasmic tyrosine kinase domains of two receptor molecules (7). The activation of the pathway depends not only on EGF as the ligand binding to the EGFR ectodomains, but also on the activation of homodimerized or heterodimerized cytoplasmic domains of EGFRs $(8,9)$. Bill et al have identified cytohesins as conformational activators of the cytoplasmic dimer, which play an important role in lung cancer ErbB signaling (10).

The cytohesin family includes four highly homologous members: Cytohesin-1, -2 (ARNO), -3 (Grp1) and -4 (11). Cytohesins are guanine nucleotide exchange factors (GEFs) for ADP ribosylation factors (ARFs) that belong to the family of small Ras-like GTPases. As with the case of other small GTPases, ARF function critically depends on activation by GEFs (12). Therefore, cytohesins are important regulators of 


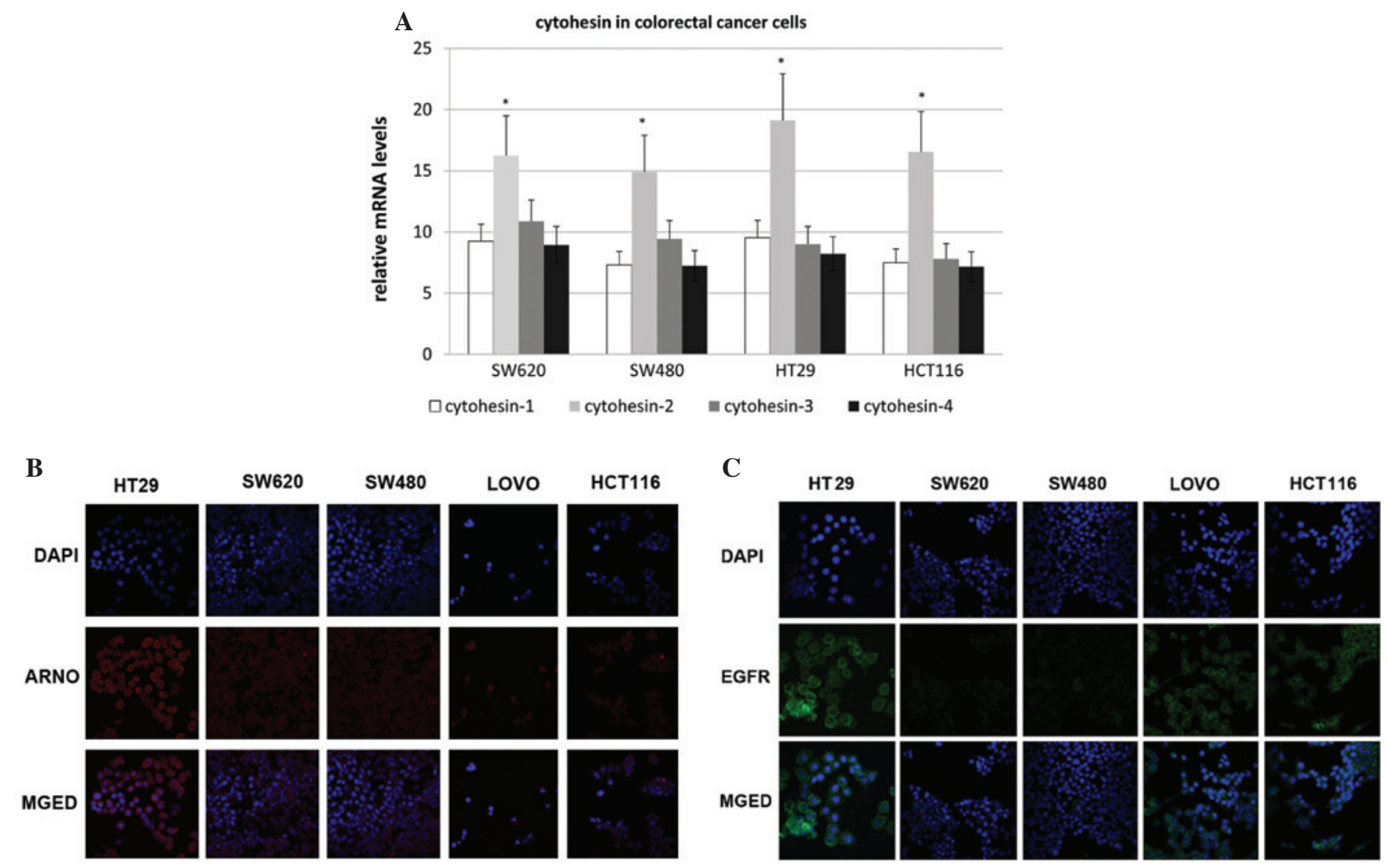

Figure 1. (A) The expression of cytohesin-1, -2 (ARNO), -3 and -4 mRNA in the four colorectal cancer cell lines. "P<0.05 for cytohesin- 2 vs. other cytohesins; $\mathrm{n}=3$. (B) The expression of ARNO in colorectal cancer cells as detected by immunofluorescence experiments. ARNO expression is evident in the HT-29, SW620, SW480, LOVO and HCT-116 cell lines. The expression of ARNO is greatest in HT-29 cells (x200). (C) The expression of EGFR in colorectal cells as detected by immunofluorescence. EGFR expression is demonstrated in the HT-29, SW620, SW480, LOVO and HCT-116 cell lines. EGFR expression is greatest in HT-29 cells. Nuclei were stained with DAPI (x200).

cytoskeletal dynamics, cell migration, vesicular traffic and signaling $(10,11,13)$.

Bill et al demonstrated that cytohesin overexpression increases EGFR activation and signaling. Moreover, siRNA and chemical inhibition of cytohesins produced consistent results both in vivo and in vitro in human lung adenocarcinomas. Therefore, the authors concluded that cytohesins were conformational activators of the ErbB receptor in lung cancer (10). In the present study, we demonstrated that EGFR signaling was reduced when cytohesins were inhibited in the HT-29 cell line. Subsequently, whether cytohesins have the potential to act as a target for colorectal cancer therapy was preliminarily investigated.

\section{Materials and methods}

Reagents. Cell culture media included RPMI-1640, McCoy's $5 \mathrm{~A}$ and L-15, which were purchased from Genom (Shanghai, China). The following mouse anti-human antibodies were used: Cytohesin-2 (cat. no. ab56510; Abcam, Hong Kong, China); p-EGFR (pY1068, cat. no. 1138-1; Epitomics, Burlingame, CA, USA); p-ERK1/2 (T202/Y204, cat. no. BS5016; Bioworld Technology, Inc., St. Louis Park, MN, USA); EGFR (cat. no. 3197; Cell Signaling Technology, Inc., Danvers, MA, USA); GAPDH (cat. no. AP0063; Bioworld Technology,
Inc.); phycoerythrin (PE)-conjugated rabbit anti-mouse $\operatorname{IgG}$ and fluorescein isothiocyanate (FITC)-conjugated goat antirabbit IgG (cat. no. GAM007; Multisciences, China). TRIzol RNA Isolation and M-MLV RTase kits were purchased from Promega Corporation (Madison, WI, USA), and the Real-Time PCR kit was purchased from Fermentas (USA). SecinH3 (cat. no. 565725/sc-203260) was purchased from Merck and siRNA oligo was purchased from Shanghai Gene Pharma (China). The following reagents, 3-(4,5-dimethylthiazol-2-yl)-2,5-diphenyl tetrazolium bromide (MTT; cat. no. m5655), dimethyl sulfoxide (DMSO; cat. no. D5879) and 0.25\% trypsin, were purchased from Sigma (St. Louis, MO, USA). Human EGF (cat. no. AF-100-15) was purchased from Peprotech, Inc. (Rocky Hill, NJ, USA) and fetal bovine serum (FBS) was purchased from Gibco (Carlsbad, CA, USA).

Cell lines and cultivation. Human colorectal cancer cell lines including HT-29, SW620, SW480, LOVO and HCT-116, were obtained from the Key Laboratory of Cancer Prevention and Intervention, Cancer Institute, Second Affiliated Hospital, School of Medicine, Zhejiang University, China. The HT-29 cell line was cultured in RPMI-1640 (with 10\% FBS and $1 \%$ streptomycin/penicillin); SW620, SW480 and LOVO cell lines were cultured in L-15 (with 10\% FBS and 1\% streptomycin/penicillin); HCT-116 cell line was cultured 
A

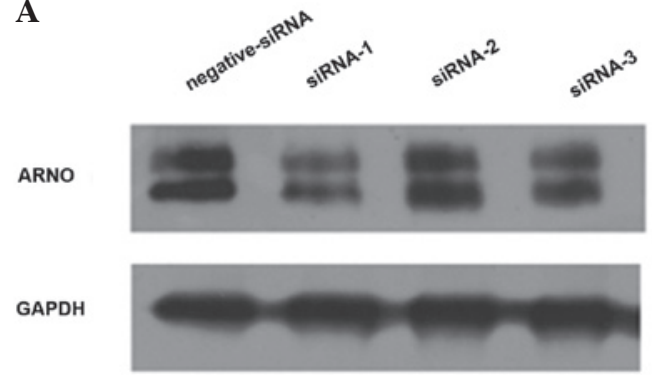

B

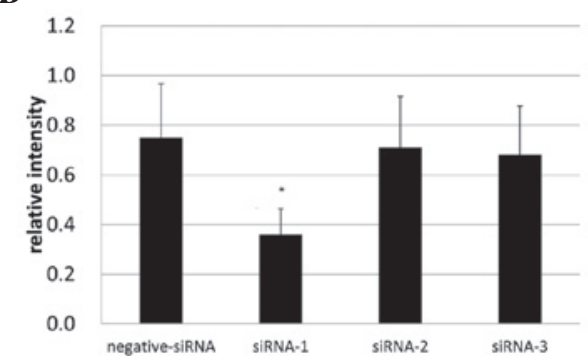

Figure 2. The expression of ARNO in HT-29 cells as detected following RNA interference of siRNA. (A) The expression of ARNO as detected by western blot analysis, following RNA interference in HT-29 cells. (B) Quantification of the trace density value of western blot analysis in HT-29 cells. ${ }^{\text {P }}<0.05$ for siRNA-1 vs. siRNA-2 or $-3 ; n=3$.

A

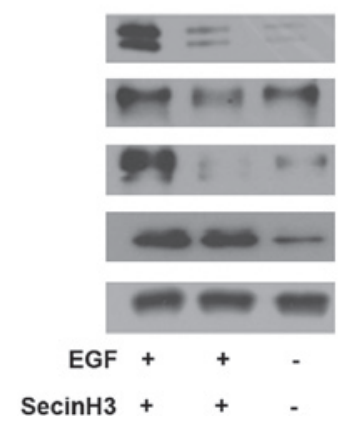

B

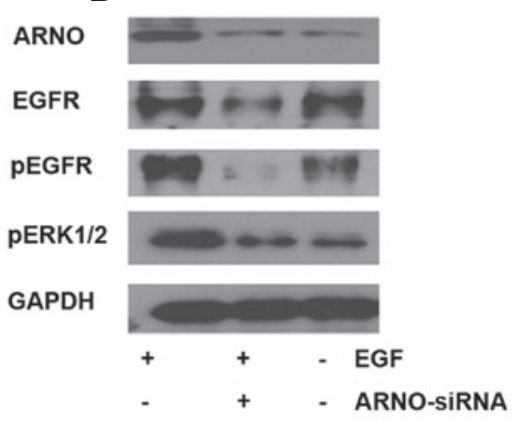

Figure 3. Cytohesin-2 (ARNO) enhances the activation of EGFR. (A) The cytohesin inhibitor SecinH3 reduces EGFR signaling. Western blot analysis of HT-29 cells treated with SecinH3 or solvent and stimulated with EGF, respectively, is shown. Phosphorylation of the indicated proteins was determined by immunodetection using phosphospecific antibodies Glyceraldehyde phosphate dehydrogenase (GAPDH) served as a loading control. (B) ARNO siRNA reduces EGFR signaling. Western blot analysis of HT-29 cells treated with ARNO-siRNA or Lipofectamine 2000 and stimulated with EGF, respectively, is shown.

in McCoy's 5A (with 10\% FBS and 1\% streptomycin/penicillin). All cell lines were cultured at $37^{\circ} \mathrm{C}$ and $5 \% \mathrm{CO}_{2}$ in an incubator, and passaged with $0.25 \%$ trypsin (Sigma) in $0.2 \mathrm{~mol} / \mathrm{l}$ phosphate-buffered saline (PBS; Sigma). The study was approved by the ethics committee of the Cancer Institute, The Second Affliated Hospital, Zhejiang University School of Medicine, Hangzhou, China.

RT-PCR. Primers were designed according to the Genbank sequences and were synthesized by Shanghai Sangon (Shanghai, China). The primer sequences were as follows: Cytohesin-1, 5'-AGTGCATTAA AGCAGCCATCAG-3' and 5'-TCAGTGTCGCTTCGTGGAG-3'; cytohesin-2 (ARNO), 5'-GAAACCGAACTGCTTTGAACT-3' and 5'-CAGCCGCCTGATGGACT-3'; cytohesin-3 (Grp1), 5'-ATG AAATCCATCAAAGCCAGTA-3' and 5'-CAATCCTT CGTTTCCTCGTT-3'; cytohesin-4, 5'-GTCCATCCGAGCC AGCAT-3' and 5'-GGTAACGGGGAACAGCAAT-3'; GAPDH (human housekeeper gene), 5'-AATGTGTCCGTCGT GGATCTG-3' and 5'-CAACCTGGTCCTCAGTGTAGC-3'. Total RNA was extracted using the TRIzol RNA isolation kit and cDNA was synthesized using the M-MLV RTase kit, according to the manufacturer's instructions. For this reaction,

GAPDH acted as an inner control and was amplified in each reaction system. The reaction conditions were $95^{\circ} \mathrm{C}$ for $3 \mathrm{~min}$, 40 cycles of $95^{\circ} \mathrm{C}$ for $10 \mathrm{sec}, 62^{\circ} \mathrm{C}$ for $35 \mathrm{sec}$ and $72^{\circ} \mathrm{C}$ for $60 \mathrm{sec}$.

Immunofluorescence. Aseptic slides were placed in 24-well plates and after prewarming at $37^{\circ} \mathrm{C}$ for $24 \mathrm{~h}, 10^{4}$ cells/well from the HT-29 cell line were incubated in the plates. Cells were cultured with RPMI-1640 culture medium at $37^{\circ} \mathrm{C}$ and $5 \% \mathrm{CO}_{2}$ in an incubator until cell growth covered $60-80 \%$ of the slides. Then, the culture medium was removed and cells were fixed in $4 \%$ paraformaldehyde for $15 \mathrm{~min}$. After washing three times with PBS and $0.25 \%$ Triton X-100/TBS for 10-15 $\mathrm{min}$ at room temperature, mouse anti-cytohesins IgG were incubated overnight at $4^{\circ} \mathrm{C}$. Following repeated washing with PBS, slides were incubated with PE-conjugated rabbit anti-mouse IgG and FITC-conjugated goat anti-rabbit $\mathrm{IgG}$ as secondary antibodies for $1 \mathrm{~h}$ at $37^{\circ} \mathrm{C}$, then washed with PBS and coverslipped. Subsequently, ARNO and EGFR expression was observed using a Zeiss LSM-710 fluorescent microscope with a Spot digital camera (Carl Zeiss, Germany). For comparable analysis of the intensity levels of ARNO and EGFR expression, the same exposure conditions were maintained throughout the experiment.

Western blot analysis. Cells were collected and extracted by the eukaryotic cell lysis buffer according to the manufacturer's instructions (Total protein extraction kit 2140, Merck Millipore, Billerica, MA, USA). Then, proteins were separated by $12 \%$ SDS-PAGE and blotted to a nitrocellulose membrane by a wet transfer device (Bio-Rad, Hercules, CA, USA). Blotted membranes were blocked by $10 \%$ skimmed milk in PBS Tween-20 (PBST) for $1 \mathrm{~h}$. After washing three times with Tris-buffered saline Tween-20 (TBST), membranes were incubated with primary antibody diluted 1:1,000 at room temperature for $1 \mathrm{~h}$, then incubated in HRP-labeled secondary antibody diluted 1:10,000 at room temperature for $1 \mathrm{~h}$. After rinsing, visualization was conducted using the enhanced ehemiluminescence (ECL) western blotting detection system (Amersham Biosciences, Little Chalfont, UK) and cells were exposed to X-ray film (Kodak, USA). GAPDH protein was used as an inner control.

siRNA selection. Three pairs of ARNO siRNAs were designed and synthesized by Genepharma Company (China). The 

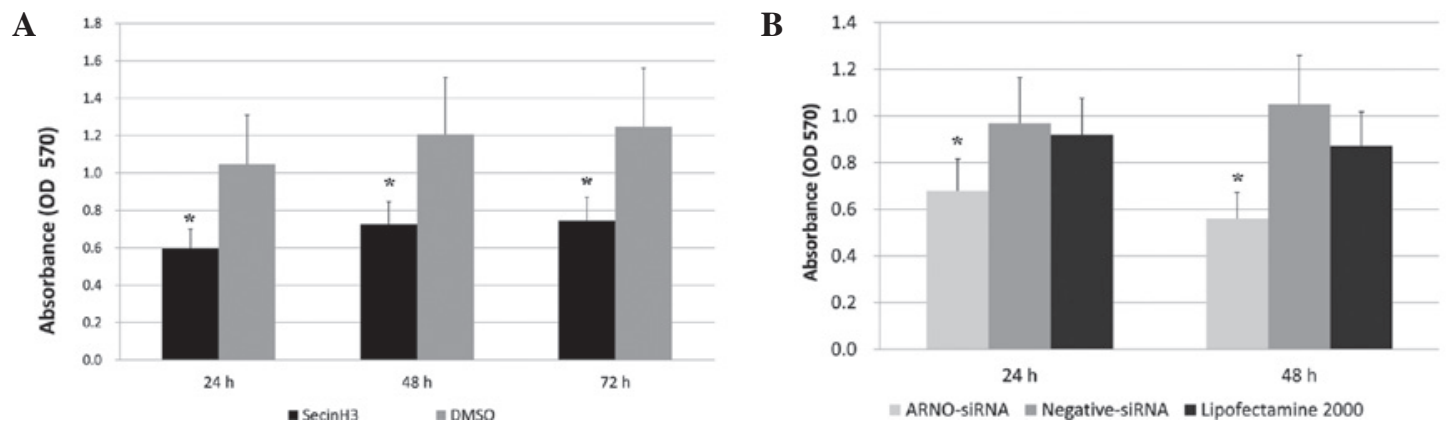

Figure 4. SecinH3 and ARNO siRNA inhibit the proliferation of HT-29 cells. (A) SecinH3 inhibits the proliferation of HT-29 cells. The graph shows the relative cell number (from an MTT assay) after 24, 48 and $72 \mathrm{~h}$ treatment with either SecinH3 or DMSO. ${ }^{*} \mathrm{P}<0.05$ for the SecinH3 blocking group vs. the control group; $\mathrm{n}=3$. (B) ARNO siRNA inhibits the proliferation of HT-29 cells. The graph shows the relative cell number (from an MTT assay) following 24 and $48 \mathrm{~h}$ treatment with ARNO siRNA or negative siRNA. "P<0.05 for ARNO siRNA vs. negative siRNA at $48 \mathrm{~h} ; \mathrm{n}=3$.

siRNA sequence pairs were as follows: siRNA-1, 5'-GUUCU UGGUGGAGAAUGAATT-3' and 5'-UUCAUUCUCCACC AAGAACTT-3'; siRNA-2, 5'-AGGCCCUCAGGCAGUUU CUTT-3' and 5'-AGAAACUGCCUGAGGGCCUTT-3'; siRNA-3, 5'-GCUGGUUUAUCCUCACAGATT-3' and 5'-UCUGUGAGGAUAAACCAGCTT-3'. Each pair of siRNA sequences was identified in the HT-29 cell line; cells were transfected with $100 \mathrm{pmol}$ of each siRNA in $5 \mu 1$ Lipofectamine $2000 / 10^{5}$ cells $/ \mathrm{ml}$, and then cultured in serumfree medium. After $24 \mathrm{~h}$, cells were collected for western blot analysis.

MTT. HT-29 cells were plated in 96-well plates with a density of 3,000 cells/well. Cells were cultured with $1 \%$ FBS and inhibitors $(20 \mu \mathrm{mol} / 1 \mathrm{SecinH} 3$ or $50 \mathrm{nmol} / \mathrm{l}$ per $5 \mathrm{pmol} \mathrm{ARNO} /$ negative siRNA in $0.25 \mu \mathrm{l}$ Lipofectamine 2000) for 24, 48 and $72 \mathrm{~h}$, at $37^{\circ} \mathrm{C}$ and $5 \% \mathrm{CO}_{2}$. Then $5 \mathrm{mg} / \mathrm{ml}$ MTT $(20 \mu \mathrm{l})$ was added to each well and incubated for $4 \mathrm{~h}$. Then, $200 \mu \mathrm{l}$ DMSO was added to resolve the MTT substrate and absorbance was measured at $570 \mathrm{~nm}$ using a SpectraMax Microplate Reader (Bio-Rad).

Statistics. Results are presented as the mean \pm standard error of the mean (SEM). The Statistical Package for the Social Sciences (SPSS) 16.0 software (SPSS, Inc., Chicago, IL, USA) was used for statistical analysis. Paired comparisons were performed using a Student's $\mathrm{t}$-test. $\mathrm{P}<0.05$ was considered to indicate a statistically significant difference between means.

\section{Results}

All four cytohesins were transcribed and ARNO was expressed in colorectal cancer cells. RT-PCR was employed to detect the transcription of the cytohesin family. Cytohesin-1,-2 (ARNO), -3 (Grpl) and -4 were transcribed in all four cell lines, which included HT-29, SW620, SW480 and HCT-116. We found that mRNA of the four cytohesins was transcribed in all four cell lines, and ARNO mRNA had the highest expression level (Fig. 1A). Additionally, by an immunofluorescence assay, we demonstrated that ARNO was highly expressed in HT-29 cells and was located in the cytoplasm, near to the membrane (Fig. 1B). Therefore, the expression of EGFR in colorectal cells was detected by immunofluorescence (Fig. 1C). The expression of EGFR in HT-29 cells was higher than that of the other cell lines. Therefore, the HT-29 cell line was selected for EGF pathway research in the following study.

siRNA-1 with the strongest inhibitory effects was selected for ARNO blocking. To select the most effective siRNA for ARNO, three siRNAs (siRNA-1, -2 and -3 ) were designed to inhibit the expression of ARNO. Expression of ARNO was then detected under the inhibition of these three siRNAs. The greatest inhbitory effect was produced by siRNA-1; the maximum inhibition rate was $49.271 \%$. Therefore, siRNA-1 was selected to be the ARNO siRNA inhibitor that was used in the present study (Fig. 2). The selected ARNO siRNA sequence pair was: 5'-AGTGCATTAAAGCAGCCATCAG-3', and 5'-TCAGTGTCGCTTCGTGGAG-3'.

Inhibition of cytohesins reduces EGF pathway signaling in HT-29 cells. To detect the function of cytohesins in the EGF pathway, cytohesins were inhibited by SecinH3 and ARNO siRNA in HT-29 cells. In the assay, HT-29 cells were cultured in $35 \mathrm{~mm}$ glass-bottom dishes, marked as group A, B or C. All cells were cultured with $1 \%$ FBS culture medium. SecinH3 (or a mixture of 100 pmol ARNO siRNA in $5 \mu$ l Lipofectamine 2000) was added to dishes from group B when cells had spread to cover $70 \%$ of the dishes for $10 \mathrm{~h}$. Simultaneously, $0.2 \%$ DMSO (or $5 \mu 1$ Lipofectamine 2000) was added to dishes from groups $\mathrm{A}$ and $\mathrm{C}$ as a control; then $50 \mathrm{ng} / \mathrm{ml}$ EGF (Peprotech, Inc.) was added to dishes from groups A and B for 5 min. Western blot analysis was employed to test the expression of the EGF pathway-associated molecules, which included ARNO, EGFR, p-EGFR and p-ERK1/2. The results indicated that when cytohesins were blocked by SecinH3 or inhibited by ARNO siRNA, ARNO expression was reduced in HT-29 cells. Additionally, phosphorylated molecules of the EGF pathway, including p-EGFR and p-ERK1/2, were downregulated in HT-29 cells (Fig. 3).

Blocking cytohesins inhibits the proliferation of HT-29 cells. To detect whether cytohesins are involved in the proliferation of HT-29 cells, we used the specific cytohesin antagonist SecinH3 and the EGFR-expressing human colorectal adenocarcinoma-derived HT-29 cells. HT-29 cells were treated with $\mathrm{SecinH} 3$ and then proliferation was detected by an MTT assay. DMSO was added to the cell culture medium in the control group. After culture for 24,48 and $72 \mathrm{~h}$, the inhibition rates of 
SecinH3 compared with the control group were 56.77, 58.72 and $57.22 \%$, respectively ( $\mathrm{n}=3$, Fig. $4 \mathrm{~A})$.

The ARNO siRNA described previously was used as an inhibitor to identify whether ARNO downregulation is capable of reducing the proliferation of HT-29 cells. The MTT assay results demonstrated that the growth and proliferation of tumor cells were significantly inhibited by ARNO siRNA at 24 and $48 \mathrm{~h}$, while the inhibition rates were 68.63 and 58.95\%, respectively, compared with the Lipofectamine 2000 group $(\mathrm{n}=3$, Fig. 4B).

\section{Discussion}

Growth and survival of cancer cells is critically dependent on specific signaling molecules (14). The EGF pathway is considered to be the most prominent signaling pathway in colorectal cancer, as it regulates the differentiation, survival, proliferation and migration of cancer cells. Recently, certain individuals with wild-type Kras gene colorectal cancer have benefited from therapies targeting the EGFR. However, resistance to the EGFR blockade inevitably occurs due to a mutation in the gene encoding EGFR that impairs the binding of cetuximab to EGFR (15-17). Therefore, it is necessary to select new targets in this pathway to overcome the resistance acquired due to mutations.

Recently, Yonesaka et al identified acquired resistance to EGFR target therapy via increased signaling through Her2 (ErbB2; also a member of the ErbB family). Notably, the authors demonstrated that either amplification of ErbB2 or increased levels of the ErbB3/ErbB4 ligand heregulin led to de novo or acquired cetuximab resistance (18), and Ruan et al achieved similar results in a breast cancer study (19). Cytohesins, family members of GTPases, have been researched for their regulation of the reassembly of the cytoskeleton and the activation of integrin or the integrin signaling system, which is critically associated with cell adhesion and migration $(11,20,21)$. A further study identified that cytohesins as EGFR activators may form a layer of positive regulation by facilitating the structural rearrangements required to convert the receptor dimer into its active conformation in lung cancer (10).

Studies by Kolanus (11) and Ogasawara et al (22) concerning the expression of cytohesins demonstrated that cytohesin-2 (ARNO) and -3 (Grpl) were ubiquitously expressed, whereas cytohesins-1 and -4 were primarily leukocyte-specific. Cytohesin-1 is a key regulator of neutrophil adhesion to endothelial cells and to components of the extracellular matrix, which may influence cell emigration through its dual opposing effect on $\beta 1$ and $\beta 2$ integrin activation (23). Additionally, ARNO behaves as a bistable switch, as it has an absolute requirement for activation by an Arf protein but, once triggered, it becomes highly active through the positive feedback effect of Arf1-GTP. This property of ARNO may provide an explanation for its function in signaling pathways that, once triggered, must move forward decisively (24). Additionally, in the present study, we detected the presence of cytohesins in the cytoplasm (near the membrane) by immunofluorescence, and ARNO was the most highly expressed cytohesin family member. Therefore, we employed molecular ARNO and the HT-29 cell line as subjects for the other sections of our study. Whether the strong expression of ARNO in colorectal cancer cells, potentially by enhanced EGFR signaling, contributes to tumor differentiation, survival, proliferation and migration, is yet to be determined. However, this has been identified in other types of cancer cells $(25,26)$.

In the cell proliferation section of the present study, HT-29 cells were stimulated by human EGF in the presence of SecinH3 or treated with ARNO siRNA. As a result, both SecinH3- and ARNO siRNA-treated cells demonstrated a 56.77-68.63\% inhibition rate compared with solvent-treated samples. Therefore, we hypothesize that inhibiting cytohesins contributed to the reduction in EGFR signaling. To identify the mechanism of this inhibition in HT-29 cells, i.e. whether the enhancement of EGFR activation by cytohesins was due to the effect of cytohesins on EGFR, we investigated the activation of certain EGFR pathway molecules (p-EGFR and p-ERK1/2). Our results gave support to this mechanism of inhibition.

In conclusion, ARNO, an important isoform of the cytohesin family, is highly expressed in colorectal cancer cells and enhances EGFR signaling, which contributes to tumor differentiation, survival and proliferation.

\section{Acknowledgements}

This research was supported by the National High Technology Research and Development Program of China (No. 2012AA02A506), the NSFC (No. 30901741), Zhejiang the Provincial Key Scientific and Technological Research Projects of International Cooperation (No. 2009C14010) and the Zhejiang Provincial Natural Science Foundation of China (No. R209.353). The authors would like to thank Dr Zhang Jiawei, Dr Fu Xianhua and Dr Wang Zhanhuai for their cell lines (HT-29, SW620, SW480, LOVO and HCT-116), support and enthusiasm.

\section{References}

1. Jemal A, Bray F, Center MM, Ferlay J, Ward E and Forman D: Global cancer statistics. CA Cancer J Clin 61: 69-90, 2011.

2. Edwards BK, Ward E, Kohler BA, Eheman C, Zauber AG, Anderson RN, Jemal A, Schymura MJ, Lansdorp-Vogelaar I, Seeff LC, van Ballegooijen M, et al: Annual report to the nation on the status of cancer, 1975-2006, featuring colorectal cancer trends and impact of interventions (risk factors, screening, and treatment) to reduce future rates. Cancer 116: 544-573, 2010.

3. Mitry E, Bouvier AM, Esteve J and Faivre J: Benefit of operative mortality reduction on colorectal cancer survival. Br J Surg 89: 1557-1562, 2002.

4. Mayer RJ: Targeted therapy for advanced colorectal cancer more is not always better. N Engl J Med 360: 623-625, 2009.

5. Siena S, Sartore-Bianchi A, Di Nicolantonio F, Balfour J and Bardelli A: Biomarkers predicting clinical outcome of epidermal growth factor receptor-targeted therapy in metastatic colorectal cancer. J Natl Cancer Inst 101: 1308-1324, 2009.

6. Bublil EM and Yarden Y: The EGF receptor family: spearheading a merger of signaling and therapeutics. Curr Opin Cell Biol 19: 124-134, 2007.

7. Bose R and Zhang X: The ErbB kinase domain: structural perspectives into kinase activation and inhibition. Exp Cell Res 315: 649-658, 2009.

8. Jura N, Endres NF, Engel K, Deindl S, Das R, Lamers MH, Wemmer DE, Zhang X and Kuriyan J.: Mechanism for activation of the EGF receptor catalytic domain by the juxtamembrane segment. Cell 137: 1293-1307, 2009.

9. Red Brewer M, Choi SH, Alvarado D, Moravcevic K, Pozzi A, Lemmon MA and Carpenter G: The juxtamembrane region of the EGF receptor functions as an activation domain. Mol Cell 34: 641-651, 2009. 
10. Bill A, Schmitz A, Albertoni B, Song JN, Heukamp LC, Walrafen D, Thorwirth F, Verveer PJ, Zimmer S, Meffert L, Schreiber A, et al: Cytohesins are cytoplasmic ErbB receptor activators. Cell 143: 201-211, 2010.

11. Kolanus W: Guanine nucleotide exchange factors of the cytohesin family and their roles in signal transduction. Immunol Rev 218: 102-113, 2007.

12. Bos JL, Rehmann $\mathrm{H}$ and Wittinghofer A: GEFs and GAPs: critical elements in the control of small G proteins. Cell 129: $865-877,2007$.

13. Casanova JE: Regulation of Arf activation: the Sec7 family of guanine nucleotide exchange factors. Traffic 8: 1476-1485, 2007.

14. Weinstein IB: Cancer. Addiction to oncogenes - the Achilles heal of cancer. Science 297: 63-64, 2002.

15. Bardelli A and Jänne PA: The road to resistance: EGFR mutation and cetuximab. Nat Med 18: 199-200, 2012.

16. Montagut C, Dalmases A, Bellosillo B, Crespo M, Pairet S, Iglesias M, Salido M, Gallen M, Marsters S, Tsai SP, Minoche A, et al: Identification of a mutation in the extracellular domain of the Epidermal Growth Factor Receptor conferring cetuximab resistance in colorectal cancer. Nat Med 18: 221-223, 2012.

17. Vlacich G and Coffey RJ: Resistance to EGFR-targeted therapy: a family affair. Cancer Cell 20: 423-425, 2011.

18. Yonesaka K, Zejnullahu K, Okamoto I, Satoh T, Cappuzzo F, Souglakos J, Ercan D, Rogers A, Roncalli M, Takeda M, Fujisaka Y, et al: Activation of ERBB2 signaling causes resistance to the EGFR-directed therapeutic antibody cetuximab. Sci Transl Med 3: 99ra86, 2011.
19. Ruan SQ, Wang SW, Wang ZH and Zhang SZ: Regulation of HRG- $\beta 1$-induced proliferation, migration and invasion of MCF-7 cells by upregulation of GPR30 expression. Mol Med Report 6: 131-138, 2012.

20. El Azreg MA, Garceau V and Bourgoin SG: Cytohesin-1 regulates fMLF-mediated activation and functions of the $\beta 2$ integrin Mac-1 in human neutrophils. J Leukoc Biol 89: 823-836, 2011.

21. Oh SJ and Santy LC: Differential effects of cytohesins 2 and 3 on $\beta 1$ integrin recycling. J Biol Chem 285: 14610-14616, 2010.

22. Ogasawara M, Kim SC, Adamik R, Togawa A, Ferrans VJ, Takeda K, Kirby M, Moss J and Vaughan M.: Similarities in function and gene structure of cytohesin-4 and cytohesin-1, guanine nucleotide-exchange proteins for ADP-ribosylation factors. J Biol Chem 275: 3221-3230, 2000.

23. El Azreg MA and Bourgoin SG: Cytohesin-1 regulates human blood neutrophil adhesion to endothelial cells through $\beta 2$ integrin activation. Mol Immunol 48: 1408-1416, 2011.

24. Stalder D, Barelli H, Gautier R, Macia E, Jackson CL and Antonny B: Kinetic studies of the Arf activator Arno on model membranes in the presence of Arf effectors suggest control by a positive feedback loop. J Biol Chem 286: 3873-3883, 2011.

25. Chardin P, Paris S, Antonny B, Robineau S, Béraud-Dufour S, Jackson CL and Chabre M: A human exchange factor for ARF contains Sec7- and pleckstrin-homology domains. Nature 384: 481-484, 1996.

26. D'Souza-Schorey $C$ and Chavrier P: ARF proteins: roles in membrane traffic and beyond. Nat Rev Mol Cell Biol 7: 347-358, 2006. 